\title{
Influence of Upsetting Process on Continued Electric Heating by Coupled Electro-thermal-mechnical Simulation
}

\author{
Zheng-xing Men, Ya-xin*Ma， Tai-wen Yue, Rui-lin Liu and Yue Tang \\ Aeronautical Manufacturing Engineering Department, Chengdu Aeronautic Polytechnic , Sichuan \\ Chengdu 610021, China
}

\begin{abstract}
Keywords: Resistance Heating, Temperature Distribution, Contact Resistance, Electro-thermo-mechanical Simulation.
\end{abstract}

\begin{abstract}
In the present study, an axis-symmetric electro-thermo-mechanical model has been developed to analyze a deformation process by continuous resistance heating. The influences of the contact resistance, the current intensity and the die geometry on the temperature distribution were investigated, and the variations of the billet temperature distribution, forming force were obtained. The simulation results correspond well with experimental measured values. Furthermore, the influence of a current increasing during forming on the billet temperature and forming force was predicted in order to optimize the forming technology by continuous resistance heating.
\end{abstract}

\section{Introduction}

The traditional hot forming technology is difficult to satisfy the real time demand because of high energy consumption, low material utilization and long production cycle. On the other hand, new material get a still higher demand on the metal forming technology because of the special properties of temperature sensitive, narrow forming range and complex phase transition during forming. It has been revealed that the application of current during metal deformation process can reduce deformation stress, improve mechanical properties and change microstructure. The troditional forming technology and the continouos heating technology has been combined. It have advantages of low forming force, low energy consumption, and can avoid the increase of produation cost due to repeated heating.

The hot forming technology by continuous resistance heating can heating the billet to the forming temperature in the die prior forming by the resistance heating and continue heating it during forming. The resistance heating is the ideal continuous heat source for it has characters such as high heat efficiency, simple device, convenient control and so on. The more important thing is which can improve the metal plastic deformation by the exist of current field. However, the forming process by resistance heating is complex, which include heat transfer theory, material science, computer control technology and so on. Many influencing factors and the complex relations each other severely obstructed the further development of the hot forming technology by continuous resistance heating. As the rapid heating rate and simple equipments, resistance heating is widely applied during the metal deformation, such as the electric upsetting [1], the resistance sintering technique [2] and the mushy state forming by resistance heating [3]. However, the deformation process with continuous resistance heating is complex due to the fact that in the process exist coupled interactions between electrical, thermal and mechanical phenomena. Therefore, research by experiments alone is difficult. In order to develop and optimize the technology of resistance heating during deformation process, the simulation of the coupled electro-thermo-plastic process is necessary.

In this study, a two-dimensional axis-symmetric electro-thermo-plastic FEM model was developed in ABAQUS to investigate the deformation process with resistance heating. It is hard to further study with traditional experiments because the continuous resistance heating forming process is a nonlinear complex of electric conduction, thermal conduction and plastic deforming. The coupling simulation of electric, thermal and force can not be inplemented at present. This article separately calculate the electric-thermal coupling and thermal-force coupling and then calculate sequential coupling of them 
and finally build the finite element model of electric-thermal-force coupling for the continuous resistance heating forming. Comparing the results of simulation and experiments, contacting resistance model built in electric-thermal coupling effectively reflect influence of contacting resistance to temperature, in which the resistivity has an inverse relation with temperature of contact surface. The veracity of math model has been validated and the distribution of temperature, current density and stress and strain has been obtained. The results have provided contion to complicated part's continuous resistance heating forming and further optimization.

\section{Numerical modeling}

Physical procedure. The experimental set-up is shown schematically in Fig. 1. The billet is placed between the dies and heated with a constant continuous current flowing through the dies and the billet due to the Joule heating effect. The billet is heated up to about $1000{ }^{\circ} \mathrm{C}$ in the dies, then kept at that temperature for about 5 seconds, and subsequently upset with a constant speed at $1 \mathrm{~mm} / \mathrm{s}$. The current is maintained during the forming process until the maximal force reaches $30 \mathrm{KN}$.

In the experiment, the cylindrical billet dimensions are: $\mathrm{d}=6 \mathrm{~mm}, \mathrm{~h}=8 \mathrm{~mm}$. The die dimensions are: $\mathrm{d}=120 \mathrm{~mm}, \mathrm{~h}=30 \mathrm{~mm}$. The material of the billet and the die is $42 \mathrm{CrMo} 4$ and TZM, respectively. The temperature during heating and forming is measured by using a K-type thermocouple positioned in the center of the billet.

Contact resistance model. During the resistance heating, the main heating increase due to Joule heating. There are two contributions to Joule heating, the billet bulk resistance heating $\mathrm{Rb}$ and the contact resistance between the die and the billet Rc. Rb is well defined with other material properties. However, the definition of Rc is difficult because it is sensitive on temperature, contact force and interface quality (roughness, hardness, pollution, oxidation and so on) [4-6].

Finite element model. In the numerical model, the mechanical status (stress and strain distribution, the billet shape, etc) are calculated by one completed thermal-mechanical analysis, which is divided at every small increment by the order restart in ABAQUS[7]. The heating temperature distribution of every increment due to the Joule heating is calculated by many electrical-thermal analyses[8]. At the beginning of the process the initial temperature field is first calculated by an electrical-thermal analysis, and then it is imposed in the thermal-mechanical analysis to calculate the mechanical status at a small increment.

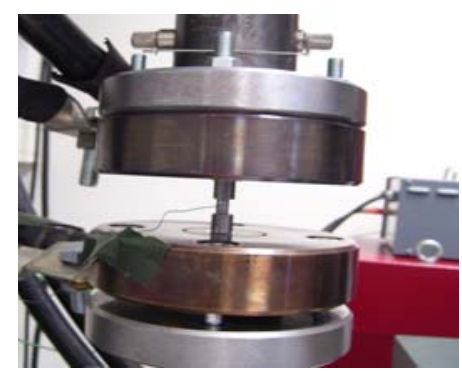

Fig. 1. Schematic set-up for forming process by continuous resistance heating

The two-dimensional axis-symmetric model to simulate the heating and forming process is developed as it shown in Fig. 2. In the electrical-thermal analysis, For the analysis, several assumptions are adopted to simplify the calculation and decrease the calculation time: (1) a direct current (DC) is applied directly at the top of the upper die, and the electric potential is zero at the bottom of the lower die instead of the function of the electrodes; (2) the die dimensions are decreased to: $\mathrm{d}=18 \mathrm{~mm}, \mathrm{~h}=10 \mathrm{~mm}$. The outer surfaces of the dies are always kept at room temperature during the simulation; (3) the mechanical deformation is neglected during the heating process prior to forming; (4) the die deformation is neglected during forming. 


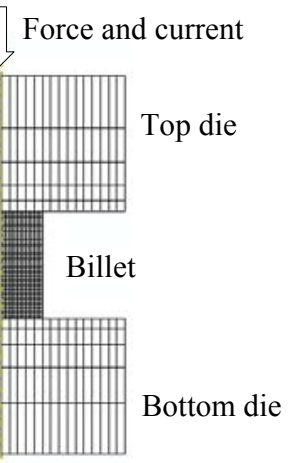

Fig. 2. Finite element model.

\section{Results and discussion}

Temperature distribution. Figure 3 is the comparison chart of the temperature change in the center of the forming process and the test results. The temperature data is obtained from the thermocouple in the center hole of the billet. From the graph we can find that the simulation results agree well with the experimental results. In the process of forming, the height of the billet is decreased, the diameter is increased, the resistance of the billet is reduced, and the heat generated by the resistance heating is reduced. The temperature field distribution of the billet at different time is shown in Figure 4. During the forming process, the heat source of billet mainly comes from Joule heat, and the heat loss is mainly through the heat conduction. Therefore, the high temperature region of the blank is located in the middle of the blank, the lowest temperature appears in the contact with the mold parts. The distribution of temperature directly affects the flow pattern of metal, it can be seen from the diagram, the flow of metal mainly occurs in the middle part of the blank.

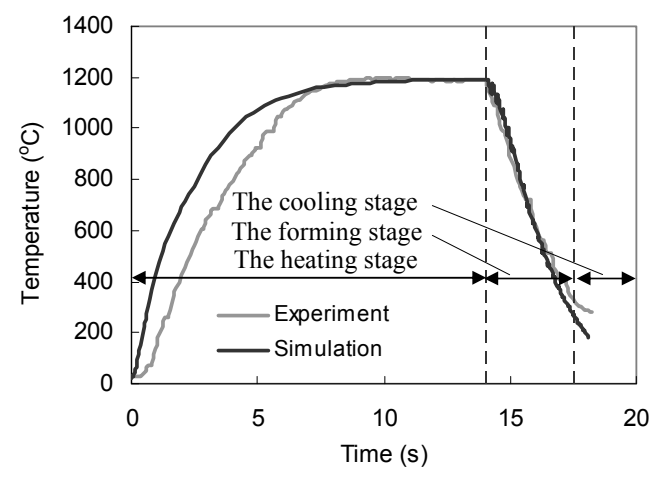

Fig. 3 Comparison of the temperature history during forming between the experiment and the simulation.

Simulation of the forming process. The temperature variation has a strong effect on the forming process. One of the major aims of the study is to investigate the variation of the temperature and forming force during the hot forming process by continuous resistance heating. In the following, the experimental data is compared with the calculation of the model. Fig. 5 shows the comparison of the temperature history of the heating and forming process between the experiment and the simulation. The experimental value was gained under the current intensity $1000 \mathrm{~A}$ and the initial contact force $0.15 \mathrm{KN}$. The whole process can be divided into three stages, namely heating, forming and cooling. It demonstrates that in two approaches, the temperature appears at the maximum value at about 10 seconds, and then declines quickly during the forming stage due to the reduction of the resistance. During upsetting the contact resistance remains at a low stage because of the higher forming force. 
Meanwhile, the billet bulk resistance decreases in according to the decrease of the billet height in the forming process.

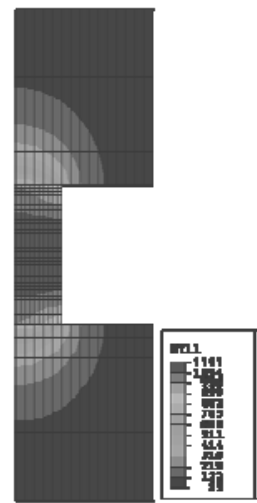

$\mathrm{T}=0 \mathrm{~s}$

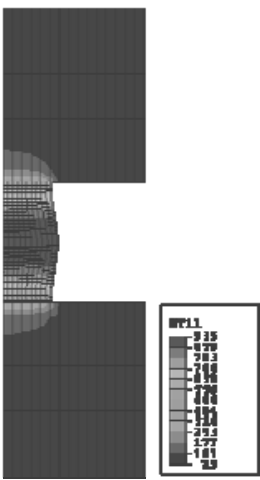

$\mathrm{T}=1 \mathrm{~s}$

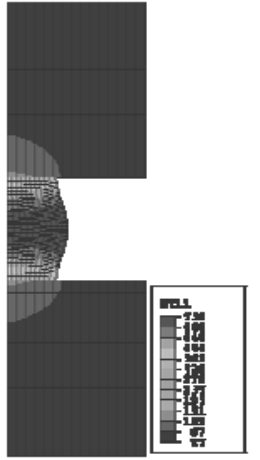

$\mathrm{T}=2 \mathrm{~s}$

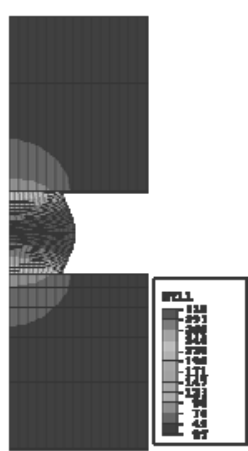

$\mathrm{T}=3 \mathrm{~s}$

Fig. 4 Temperature distribution during forming

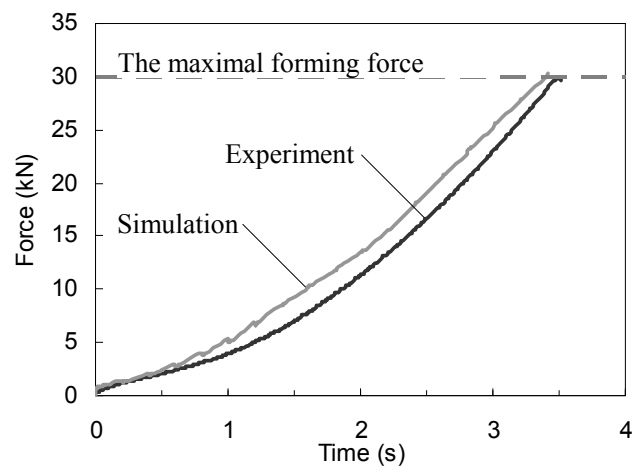

Fig. 5 Comparison of the forming force during forming between the experiment and the simulation.

Although the conformity between the simulation and the experiment is good, the model tends to overestimate the temperature value in the heating stage. One possible reason is that the decrease of the contact resistance in the simulation is slower than in the experiment. The agreement between the two approaches is satisfactory. The temperature decrease caused by the decrease of the resistance during forming leads to a significant increasing of the forming force.

The comparison between the numerical simulation and the experimental results is shown in Figure 6. With the continued resistance heating, the contact surface energy to the blank center conduction, so the blank temperature rise, and the rise of billet temperature and cause increase billet volume resistance, and lead to the increase of billet temperature, and temperature increase caused by the increase of blank volume resistance and contact resistance, contact surface due to temperature rise sharply finally, the billet volume decreased, resistance plays a dominant role in the process of heating, the center of the heat dissipation is reduced, the highest temperature appears at the center of the blank.

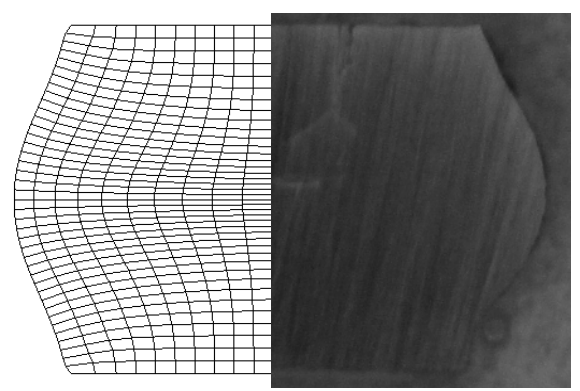

Fig. 6 Comparison of the section shape between the experiment and the simulation 
Based on the results described above, it is obvious that the electro-thermo-mechanical model is effective to simulate the forming process by continuous resistance heating. By increasing the current intensity, the decrease of the cooling rate and the forming force can be obtained.

\section{Conclusions}

An electro-thermo-mechanical model of the hot-forging process by continuous resistance heating was developed using the finite element method.

The temperature change, load and the forming result of the blank center are obtained by numerical simulation, which is consistent with the experiment, which verifies the reliability of the simulation. In the process of forming a constant current, the temperature of the billet is decreased sharply because of the decrease of the contact resistance and the volume resistance.

During the forming process, the stress and strain of the billet are mainly affected by the temperature field, and the maximum stress appears in the region with the lowest temperature, i.e., the outer edge of the blank and the contact surface of the die. The maximum equivalent stress of the billet is always in the highest temperature, that is, the central part of the workpiece.

The forming process of increasing the strength of the current in the blank cooling speed temperature center greatly decreased, the forming load is only $1 / 3$ of the load, therefore, the change of the heating current effectively improve billet temperature, improve the forming environment. However, it is difficult to determine the temperature of the billet by changing the temperature of the heating current. The method of numerical simulation is difficult to be determined, and the optimization method is needed to be further studied.

\section{References}

[1] N. Biba, A. Lishnij, A. Vlasov: Simulation of coupled problem of electric upsetting. Journal of Materials Processing Technology, 80-81 (1998) 184-187.

[2] S. Maki, Y. Harada, K. Mori: Application of resistance sintering technique to fabrication of metal matrix composite. Journal of Materials Processing Technology, 119(1-3) (1998) 210-215.

[3] Seijiro Maki, Yasunori Harada, Ken-ichiro Mori, Hiroyasu Makino: Application of resistance heating technique to mushy state forming of aluminium alloy. Journal of Materials Processing Technology, 125-126 (2002) 477-482.

[4] P. Rogeon, P.Carre, J.Costa, G.Saindrenan .: Characterization of electrical contact conditions in spot welding assembilies.Journal of Materials Processing Technology 195 (2008) 117-124.

[5] E. Feulvarch, V. Robin, J. M. Bergheau: Resistance spot welding simulation: a general finite element formulation of electrothermal contact conditions. Journal of Materials Processing Technology. 153-154 (2004) 436-441

[6] Zhigang Hou, Ill-Soo Kim, Yuanxun Wang, Chunzhi Li, Chuanyao Chen: Finite element analysis for the mechanical features of resistance spot welding process. Journal of Materials Processing Technology. 185 (2007) 160-165

[7] I. Ranjbar Nodeh, S. Serajzadeh, A. H. Kokabi: Simulation of welding residual stresses in resistance spot welding, FE modeling and X-ray verification. Journal of Materials Processing Technology. 205 (2008) 60-69

[8] P. Rogeon, P.Carre, J.Costa, G.Saindrenan .: Characterization of electrical contact conditions in spot welding assembilies.Journal of Materials Processing Technology 195 (2008) 117-124. 